\title{
SOSA EN TORNO AL CONOCIMIENTO, EL JUICIO Y LA ADIVINACIÓN
}

\author{
SOSA ON KNOWLEDGE, JUDGMENT AND GUESSING
}

\author{
J. Adam Carter
}

\section{RESUMEN}

En el capítulo 3 de Judgment and Agency, Ernest Sosa (2015) elabora el concepto de una actuación completamente apta. Al hacer esto, apela a ejemplos ilustrativos de actuaciones prácticas y aplica las lecciones aprendidas al caso de las actuaciones cognitivas y, en particular, a la actuación cognitiva que es el juicio. Los ejemplos de Sosa en la esfera práctica son ricos e instructivos. Pero, argumento, hay una falta de analogía interesante entre los ejemplos prácticos y cognitivos a los que apela. En última instancia, creo que la falta de analogía tiene su fuente tanto en una concepción problemática de la actuación cognitiva de la adivinación, así como en su relación con el conocimiento y la derrota. ${ }^{1}$ Una vez que se presenta esta línea argumentativa crítica, se articula una concepción alternativa de la adivinación, qua actuación cognitiva. Tal concepción evita los problemas discutidos y, aún así, es compatible con el marco general de Sosa.

PALABRAS CLAVE: Ernest Sosa, conocimiento, juicio, adivinación

\begin{abstract}
In Chapter 3 of Judgment and Agency, Ernest Sosa (2015) explicates the concept of a fully apt performance. In the course of doing so, he draws from illustrative examples of practical performances and applies lessons drawn to the case of cognitive performances, and in particular, to the cognitive performance of judging. Sosa's examples in the practical sphere are rich and instructive. But there is, I will argue, an interesting disanalogy between the practical and cognitive examples he relies on. Ultimately, I think the source of the disanalogy is a problematic picture of the cognitive performance of guessing and its connection to knowledge and defeat. Once this critical line of argument is advanced, an alternative picture of guessing, qua

\footnotetext{
${ }^{1}$ Nota del traductor: utilizamos el término "derrota", y sus asociados (e.g. "derrotabilidad" y "derrotante"), para traducir el término inglés "defeat", y sus asociados (e.g. "defeasibility" y "defeater").
} 
cognitive performance, is articulated, one which avoids the problems discussed, and yet remains compatible with Sosa's broader framework.

KEYWORDS: Ernest Sosa, knowledge, jugment, guessing

\section{INTRODUCCIÓN}

El tema de este artículo es un tipo particular de actuación cognitiva: la adivinación. De acuerdo con Sosa (2015), adivinar es parecido a juzgar, pero no completamente. Tanto el juicio como la adivinación tienen una finalidad alética. Cuando adivinamos, así como cuando juzgamos, lo hacemos con el propósito de dar con la verdad. El juicio tiene un propósito epistémico ulterior, que es dar con la verdad de manera apta. Y dar con la verdad de manera apta involucra una evaluación de riesgo competente.

Una evaluación de riesgo competente - y no sólo dar en el blanco relevante de cualquier manera - obviamente es importante cuando se participa en algún deporte, como el baloncesto. Supongamos que lanzas la pelota desde mucho más allá de tu umbral de competencia fiable — supongamos que arrojas la pelota hacia la canasta desde la línea de medio campo-. En el contexto de un juego de baloncesto, ésta es una elección de tiro muy pobre (asumiendo que el reloj de disparo no está por expirar). Tu entrenador y tus compañeros de equipo te reprenderán con justa razón por realizar un lanzamiento tan desatinado, incluso si el lanzamiento termina por entrar en la canasta. Algo similar sucede en la arena cognitiva: incluso si una adivinación improbable resulta correcta por casualidad —e incluso si (como en el caso del lanzamiento de medio campo) tenías el propósito de dar con la verdad mediante una adivinación, algo hace falta—. Una adivinación correcta pero improbable (tal y como el lanzamiento de medio campo que entra en la canasta) no es apta, lo cual quiere decir que su corrección no manifiesta la competencia del tirador de realizar ese lanzamiento de una manera suficientemente fiable. ${ }^{2}$

${ }^{2}$ La cuestión de qué tan fiable es "suficientemente fiable" no es invariable a través de los dominios de actuación, sino que es sensible a un rango de factores específicos de los dominios particulares. De acuerdo con Sosa (2015), 'En baloncesto sabemos más o menos en dónde se encuentra éste, tomando en consideración la posición del jugador y sus compañeros de equipo, el tiempo que queda en el reloj, si el lanzamiento a realizar es de tres puntos o no, etc. De esta manera muchos factores tienen un impacto de maneras diversas, y los buenos jugadores los tomarán en cuenta, tratando no sólo de hacer una canasta, sino también de manifestar, al 
Desde luego, en un partido de baloncesto es muy raro que se realicen lanzamientos desde el medio campo. De manera similar, al intentar responder una pregunta, es muy raro - a menos que seamos forzados por la práctica ${ }^{3}$ - que intentemos responderla correctamente mediante una adivinación improbable. ${ }^{4} \mathrm{Sin}$ embargo, compárense un lanzamiento desde medio campo (más allá del umbral de la competencia fiable propia) con un lanzamiento apenas dentro del umbral de la competencia fiable propia, aunque realizado en una circunstancia en la que no puedes determinar que estás de hecho dentro de este umbral. Este último caso es claramente diferente del caso en el que realizas un lanzamiento desde el medio campo. Tu lanzamiento en este caso es apto, correcto a causa de la destreza. Sin embargo, está presente un tipo de suerte que reduce el crédito, que afecta a la evaluación de la actuación. Puesto de manera simple, hasta donde sabías, cuando lanzaste el tiro te encontrabas fuera de tu umbral de fiabilidad. Simplemente sucedió que te encontrabas dentro de este umbral. De manera más cuidadosa: tu actuación fue segura en el primer orden, pero no segura en el segundo orden. ${ }^{5}$ Simplemente sucede que eres lo suficientemente fiable desde esa distancia de una manera tal que no sería fácil que fallaras un lanzamiento desde ahí. Pero dado que no eres consciente de dónde se encuentra este umbral, también fácilmente podrías haber realizado un lanzamiento de manera inapta, apenas más allá de tu umbral de fiabilidad suficiente.

De la misma manera que un lanzamiento en baloncesto, bajo estas circunstancias, no es completamente apto, tampoco lo es la actuación que Sosa considera como el análogo cognitivo de tal lanzamiento en baloncesto: una adivinación hecha en condiciones en las cuales te encuentras apenas sobre el umbral de fiabilidad su-

\footnotetext{
hacerlo, la competencia completa requerida" $(2015,71)$. Véase también Sosa (2015, Cap. 8), especialmente pp. 168-180, donde se encontrará una discusión más detallada de lo que implica ser suficientemente fiable, dentro del contexto de la ocupación.

${ }^{3}$ Éste sería el caso cognitivo análogo de ser forzado en la práctica a realizar un lanzamiento, como cuando el reloj de lanzamiento está por expirar. Un ejemplo en la arena cognitiva de tal situación sería aquella que enfrenta un viajero, con hambre y sediento, quien debe escoger qué camino seguir en una bifurcación, y a quien le han dicho que sólo un camino lo llevará a encontrar comida y agua. En este caso, las circunstancias prácticas fuerzan al viajero a tratar de dar con la respuesta correcta mediante una adivinación improbable. Pero, si el viajero no se encontrase bajo tal coerción, intentaría dar con la respuesta correcta, pero no mediante una adivinación improbable — sino mediante medios más fiables.

${ }^{4}$ Hacer una adivinación improbable, bajo ningún tipo de coerción, constituye una pobre elección de lanzamiento epistémico.

${ }^{5}$ Véase Sosa $(2015,72)$.
} 
ficiente, pero no te das cuenta de que esto es así. Tal adivinación (a diferencia de una adivinación improbable) sería segura en el primer orden. Pero no sería segura en el segundo orden dado que, sin darte cuenta de dónde se encuentra el umbral, podrías haber adivinado muy fácilmente de manera inapta. Tal adivinación sería apta, pero no completamente apta; podría aspirar a ser conocimiento animal, pero no conocimiento reflexivo. ${ }^{6}$

Lo que se ha dicho hasta aquí captura sólo en términos muy generales el pensamiento de Sosa acerca de la adivinación, y cómo la ésta se relaciona con otras nociones dentro de su epistemología de virtudes. Sosa tiene muchas otras cosas esclarecedoras que decir al respecto. De hecho, sus observaciones con respecto a la adivinación (y sus análogos, en el caso de las actuaciones prácticas) emergen en varios lugares a lo largo de Judgment and Agency. En particular, lo que Sosa dice sobre la adivinación tiene un papel importante y central en su elucidación, en el capítulo 3, del concepto - central en Judgment and Agency- de una actuación completamente apta. Esto distingue a la adivinación como un elemento importante en el proyecto amplio que Sosa defiende en el libro, dado que hace uso del concepto de una actuación completamente apta en su elucidación de la naturaleza y normatividad del juicio humano — siendo éste último su objetivo primario.

En este artículo, ofrezco una crítica que se concentra en el tratamiento que defiende Sosa de la adivinación. Presto especial atención al papel que tiene la adivinación en el proyecto dialéctico de Sosa en el capítulo 3, y en menor medida, en los capítulos 6 y 8 de Judgment and Agency. En respuesta a las objeciones que se han presentado ofrezco una manera alternativa de tratar la adivinación que evade estas objeciones y que, sin embargo, sigue siendo compatible con el marco general de la epistemología de virtudes de Sosa.

Éste es el plan. En $\ 2$ se detalla la postura de Sosa con respecto a la adivinación, tomando como punto de partida su caso del EXAMEN DE LA VISTA, el cual es un punto importante de referencia en el capítulo 3. En $\$ 3$ se introduce la noción de derrota de la actuación y se subrayan, con referencia a los derrotantes performativos, algunas diferencias estructurales entre EXAMEN DE LA VISTA y otros casos que Sosa presenta como si estuvieran a la par en términos performativos con EXAMEN DE LA VISTA. En $\$ 4$ se apela a la idea jamesiana de que la finalidad de poseer la verdad debe entenderse como una mezcla de dos finalidades que compiten entre si - representar de manera verdadera y no representar falsamen-

\footnotetext{
${ }^{6}$ Véase también Sosa (2010) para una discusión de la diferencia entre una competencia reflexiva y una de primer orden.
} 
te- para sugerir que las adivinaciones, incluso las fiables, generan derrotantes de una manera tal que (contra Sosa) las inhabilita para que sean consideradas conocimiento, animal o de otro tipo. En $\$ 5$ se discute el enfoque de la adivinación que se ha esbozado hasta ahora en el contexto más amplio de los logros no fiables, y se distinguen varios tipos de adivinaciones con relación a lo que se presenta como sus análogos en el caso de la práctica.

\section{SOSA EN TORNO A LA ADIVINACIÓN}

Sosa (2015) presenta el ejemplo siguiente:

EXAMEN DE LA VISTA: Cuando voy a mi examen de la vista, me piden leer las líneas en un gráfico que tiene letras que se encogen de una línea a otra, desde una letra enorme en la parte superior hasta aquéllas letras que son apenas visibles en la parte inferior. En algún punto empiezo a perder la confianza en que estoy leyendo correctamente las letras, pero continúo hasta que el optometrista me pide que me detenga y después registra los resultados. En ese punto hay muchos casos en los que estoy bastante inseguro de si una letra particular es una " $E$ " en lugar de una "F", por ejemplo, o una "P" en lugar de una "F", etc. Supongamos, sin embargo, que resulta que (sin saberlo) de hecho estoy infaliblemente en lo correcto año tras año en una línea donde estoy inseguro de esta manera. En ese punto efectivamente estoy adivinando. De hecho, hago estas afirmaciones, para mí mismo en privado y para el optometrista en público, y las hago con la intención de dar con la respuesta correcta. Después de todo, esto es lo que el examen requiere: que trate de responder correctamente. Ciertamente podemos estipular que de esta manera manifiesto una competencia, una que no reconozco como suficientemente fiable. Esto último es la razón por la que recurro a la adivinación cuando sigo haciendo afirmaciones conforme el examen avanza. Sin saberlo, sin embargo, mis afirmaciones resultan ser sorprendentemente fiables $(2015,74-5)$.

EXAMEN DE LA VISTA —específicamente, las circunstancias en las que uno lee de manera fiable la última línea, al tiempo que uno no es consciente de que lo hace de manera fiable - ofrece lo que podríamos llamar adivinación fiable. Es fiable porque el sujeto examinado está infaliblemente en lo correcto al leer la línea final en el examen de la vista. Pero, como nos dice Sosa, es sin embargo una adivinación; no consideras tu competencia como si fuera suficientemente fiable cuando lees la última línea, incluso cuando lo sea.

Aquí encontramos tres afirmaciones centrales que Sosa hace con respecto a la adivinación fiable. Primero, la adivinación fiable — de la misma manera que la adivinación en general - tiene una finalidad (meramente) alética; en el caso de EXA- 
MEN DE LA VISTA, el agente tiene el propósito de dar con la respuesta correcta mediante la adivinación, pero no de dar con la respuesta correcta de manera apta ${ }^{7}$. Segundo, la creencia del sujeto que adivina de manera fiable es un tipo de conocimiento animal subdóxico $(2005,76)$ : sus adivinaciones no son correctas a causa de la suerte (Ibíd., p. 76); en su lugar, el sujeto que adivina hace sus afirmaciones de una manera que manifiesta una competencia de primer orden visual-perceptiva, que, sin embargo, no es reconocida por el sujeto como suficientemente fiable. ${ }^{8}$

Tercero, ¿qué es lo que distingue al conocimiento que el sujeto examinado posee acerca de las letras grandes en la fila superior del tipo de conocimiento animal (subdóxico) que el sujeto posee acerca de las letras pequeñas en la fila inferior, un conocimiento que el sujeto posee en virtud de la adivinación fiable? Aquí la respuesta de Sosa es matizada. Primero considera, y después descarta, la posibilidad de que la diferencia epistémica relevante entre estos dos casos de una afirmación que tiene la finalidad de dar con la respuesta correcta sea simplemente que el sujeto examinado presenta sus afirmaciones con mayor confianza en el caso de las letras en la fila superior que en el caso de las letras en la fila inferior (aun cuando esto efectivamente sea asî). ${ }^{9}$

${ }^{7}$ En palabras de Sosa (2015, 75), "Hacemos afirmaciones incluso en las filas inferiores, al mismo tiempo que tratamos de dar con la respuesta correcta. Tratamos lo mejor que podemos, pues sólo de esta manera cumpliremos satisfactoriamente con el examen de la vista. ¿Pero intentamos dar con la respuesta correcta, de manera apta, de una manera suficientemente fiable? No, en ese punto importa poco si damos con la respuesta correcta de una manera fiable en absoluto ... hacemos una adivinación sin tratar de dar con la respuesta correcta de manera apta". Véase también la nota a pie de página 14 de Sosa.

${ }^{8}$ Véanse también las observaciones de Sosa en el capítulo 6, después de su discusión del caso de Simone. Allí, Sosa reitera su afirmación de que el conocimiento animal subdóxico sólo requiere de afirmación apta, y por lo tanto que ‘sólo requiere de adivinación' $(2015,151)$. La noción de Sosa de conocimiento animal subdóxico no debe confundirse con lo que Goldman y Olsson $(2009$, \$1) llaman 'conocimiento débil', el cual no tiene que ser fiable, sino sólo verdadero. Goldman y Olsson, apelando a ejemplos como aquéllos presentados por Hawthorne (2002), señalan que en algunas ocasiones es adecuado atribuir conocimiento a individuos que generan la respuesta correcta a una pregunta, donde la posesión de esta información contrasta de manera sobresaliente con la ignorancia (2009, 29-20). Como una nota adicional, quiero notar que el caso de Simone es extremadamente rico y, de muchas maneras, creo que es mucho más complejo que sus otros ejemplos; ofrezco un diagnóstico detallado de este caso en otro trabajo.

${ }_{9}$ Tal y como señala Sosa, una confianza mayor es algo que puede adquirirse mediante terapia, sin que ocurra ningún otro cambio. "Esto no nos proporcionaría el tipo de conocimiento que poseemos con respecto a las letras grandes. De hecho, este incremento artificial de la confianza puede empeorar la posición epistémica del sujeto" $(2015,76)$. Incluso si el sujeto examinado tiene una mayor confianza en el caso de las letras grandes, no puede ser esta confianza lo que explica la diferencia de las posturas epistémicas. 
En su lugar, Sosa piensa, la diferencia en el estatus epistémico debe ser explicada específicamente en términos de una diferencia en la competencia reflexiva, o de segundo orden, presente en ambos casos. Sus consideraciones a este respecto son iluminadoras:

Compárese a alguien que no sólo gana confianza, sino que también adquiere una confirmación de que sigue dando con la respuesta correcta de manera fiable incluso cuando las letras se encogen. Quizás esta sea la ganancia que lo lleva a un nivel epistémico más alto. En este punto el sujeto podría obtener el conocimiento que requiere de un juicio, no meramente de una adivinación. En tal caso su conocimiento estará compuesto no sólo por un mayor grado de confianza, sino por la meta-garantía de que, incluso en el caso de las letras muy pequeñas, su nivel de competencia circunscribe al riesgo epistémico dentro de límites apropiados. En ausencia de tal información adicional, el sujeto que se somete al examen de la vista carece de una confianza bien fundada en el segundo orden de que sus afirmaciones de primer orden son más que meras adivinaciones. Incluso si, por hipótesis, sus adivinaciones son correctas y no meramente a causa de la suerte, el sujeto no puede estar seguro de esto, no de manera competente. En contraste, cuando las letras cercanas a la parte superior son claras y discernibles de manera distintiva, no adivinamos simplemente, lo cual concuerda con nuestro conocimiento de que podemos identificar esas letras por lo que son, que nuestras afirmaciones con respecto a esas líneas serían aptas $(2015,76)$.

En resumen, el sujeto que adivina fiablemente de Sosa carece de cierta metagarantía — viz., una garantía de que su nivel de competencia (de primer orden) circunscribe al riesgo epistémico dentro de límites apropiados-. El sujeto que adivina de manera fiable no puede, ex bypothesi, determinar si sus adivinaciones serían, en caso de ser correctas, correctas a causa de la suerte.

Nótese, sin embargo, que la garantía de segundo orden de la cual carece el sujeto que adivina fiablemente (garantía de la cual no carece cuando hace las afirmaciones con respecto a las letras grandes en la parte superior) no podría ser obtenida simplemente mediante la adquisición de una mayor confianza de que sus declaraciones de primer orden son lo suficientemente fiables. ${ }^{10}$ Tal confianza podría obtenerse de manera incompetente o podría estar infundada, tal y como sería el caso si el sujeto que adivina fiablemente creyese, sobre la base de un deseo, que

\footnotetext{
${ }^{10}$ Esto es distinto a tener una mayor confianza, con respecto a cada adivinación, de que cada una es correcta.
} 
sus declaraciones de primer orden fueran fiables, incluso si de hecho lo fuesen. ${ }^{11}$ En su lugar, el sujeto que adivina fiablemente carece de conocimiento de que puede identificar esas letra por las letras que son y que por lo tanto sus declaraciones en esta linea serian aptas. Hasta donde sabe el sujeto que adivina fiablemente, no lo serían.

\section{DERROTANTES PERFORMATIVOS}

Se supone que el sujeto que adivina fiablemente de Sosa en EXAMEN DE LA VISTA está a la par en términos performativos con otros dos personajes del capítulo 3:

(i) el jugador de baloncesto (a quien se hizo referencia en \$1), quien encesta la pelota de manera apta apenas por encima de su umbral de fiabilidad. El tirador, quien tiene éxito en su empresa de encestar la pelota, no está consciente de que se encuentra por encima de su umbral de fiabilidad, y que por lo tanto muy fácilmente pudo haber hecho un lanzamiento no apto.

(ii) una cazadora que, a pesar de haber tomado mucho vino, dispara de manera apta a un conejo que huye en la bruma del crepúsculo. Sosa estipula que el disparo de la cazadora fue apto - exitoso a causa de la destreza con que fue ejecutado- a pesar de que (tal y como el sujeto que adivina fiablemente y el jugador de baloncesto) no es reflexivamente competente, porque la cazadora no está consciente de que su disparo en estas condiciones es apto y ha, por lo tanto, subestimado su proeza.

Me parece, sin embargo, que a estos tres casos —el sujeto que adivina fiablemente, el jugador de baloncesto y la cazadora - subyacen algunas diferencias sutiles, pero en última instancia importantes. Sugeriré que las diferencias importan para determinar si debemos estar de acuerdo en que el sujeto que adivina fiablemente in EXAMEN DE LA VISTA obtiene un tipo de conocimiento animal, el tipo que Sosa llama conocimiento animal 'subdóxico', inclusive si concedemos a Sosa la estipulación de que el sujeto que adivina fiablemente afirma de manera apta cuáles son las letras de la parte inferior. Y el diagnóstico de por qué esto es así nos llevará a una mejor elucidación de la adivinación (o espero mostrar que esto es asî).

Para determinar cuáles son las diferencias entre los tres casos, es importante, en primer lugar, notar una diferencia de segundo orden crucial entre el caso de la cazadora y el jugador de baloncesto. Podemos decir que el jugador de baloncesto

${ }^{11}$ Véase Sosa $(2015,75$, nota 14). 
es inconsciente en el segundo orden del umbral de su propia fiabilidad. Tal y como Sosa describe el caso, el jugador:

Lanza la pelota de manera indiscriminada desde distancias muy cercanas a su umbral de fiabilidad suficiente [...] En esa zona —apenas por encima del umbral— aún tiene una alta probabilidad de tener éxito, incluso sin que el sujeto lo sepa. A pesar de todo, incluso en su ignorancia todavía puede llevar a cabo esta actuación con aptitud animal, pero la aptitud completa se encuentra más allá de sí mismo $(2015,70)$.

La ignorancia de segundo orden con respecto a si estás por encima o por debajo de tu umbral de fiabilidad suficiente es diferente de estar equivocado en el segundo orden con respecto a tu umbral de fiabilidad suficiente, aún cuando ser ignorante, así como estar equivocado en el segundo orden acerca de tu umbral de fiabilidad suficiente, bastan para prevenir que tu empresa de primer orden sea reflexivamente competente $y$, por lo tanto, completamente apta.

El jugador de Sosa es ignorante en el segundo orden, pero para encontrar a un individuo que está equivocado en el segundo orden, simplemente tenemos que considerar de nuevo a su cazadora, Diana. Tal y como Sosa describe el cao, Diana piensa que tiene bajas probabilidades de éxito (dado que ha bebido mucho vino, y dada la bruma del crepúsculo) y, considerando esto, "subestima su proeza" (2015, 68).

A diferencia del jugador de baloncesto, Diana de hecho piensa que se encuentra fuera de su umbral de fiabilidad, aunque de hecho se encuentra dentro del mismo. El jugador de baloncesto no piensa esto. El jugador de baloncesto no subestima (ni, para el caso es lo mismo, sobreestima) su proeza, como sí lo hace Diana. Él simplemente lanza la pelota de manera apta al mismo tiempo que carece de cualquier apreciación sobre si se encuentra dentro de su umbral de fiabilidad suficiente.

La diferencia entre el jugador de baloncesto y Diana puede explicarse en términos de lo que podemos denominar derrotante performativo. ${ }^{12}$ En la discusión epistemológica tradicional acerca de los derrotantes, ${ }^{13}$ éstos son creencias o dudas que cuentan en contra (en el caso de los derrotantes que refutan) de la verdad de la creencia relevante, o (en el caso de los derrotantes que socavan) en contra de la fiabilidad de la formación de la creencia relevante.

\footnotetext{
${ }^{12}$ Cf., Carter y Navarro (2015) para un tipo muy distinto de derrotabilidad performativa, una en la que el estatus epistémico que es derrotado es conocimiento-cómo, en una interpretación anti-intelectualista del mismo.

${ }^{13}$ Véase, por ejemplo, Bergman (2005); Lackey (2008); Pollock (1986); Sudduth (2008).
} 
Llamemos a la creencia de un sujeto $S$ en $p$ un derrotante performativo si, con respecto a alguna actuación $A$, la creencia de $S$ en $p$ (en el caso en que refuta) cuenta en contra de que $S$ sea exitoso en $A$ y (en el caso en que socava) pone en duda la fiabilidad de la competencia de $S$ en $A$ indicando que si $S$ lograra el éxito en $A$, el éxito no sería apto. Formulado de esta manera, desde luego es posible que haya traslapo entre estos derrotantes. Por ejemplo, supongamos que la actuación en cuestión es la de hacer un tiro corto exitoso en golf. Supongamos además que, de manera irracional, un golfista piensa que sufre de un severo síndrome de Ehlers-Danlos, un desorden invisible, pero que debilita el tejido conectivo. La creencia del golfista de que sufre de Ehlers-Danlos es un derrotante performativo que refuta. Cuenta en contra de que logre su objetivo (hacer el tiro corto) de manera exitosa en esta ocasión de actuación. Pero también es un derrotante que socava (en el sentido descrito) porque indica que, si fuese un tiro exitoso, el éxito no sería apto — no sería exitoso gracias a la destreza del tiro sino porque la pelota terminó entrando a pesar de la no fiabilidad del tiro.

Sin embargo, los derrotantes performativos, tanto los que refutan como los que socavan, pueden darse por separado. Considérese que, tal y como han sido descritos, los derrotantes performativos son estados mentales o psicológicos: ${ }^{14}$ ejercen su función, de acuerdo con Lackey, simplemente "al ser objetos de un estado psicológico relevante", y no en virtud de que sean racionales o verdaderos. De esta manera, habrá casos en los que los dos tipos de derrotantes pueden darse por separado.

Por ejemplo, supongamos que la diestra cazadora de Sosa, Diana, es informada de que un bromista ha colocado campos de fuerza invisible alrededor de muchos, pero no todos los blancos en el campo. Supongamos además que los blancos - tanto los que tienen campos de fuerza como los que no los tienen- están lo suficientemente lejos como para que sólo alguien con una gran habilidad, tal como Diana, podrían ser capaces de dar en un blanco en este rango. $\mathrm{Al}$ adquirir la creencia de que algunos de estos blancos están rodeados por un campo de fuerza invisible, la cazadora adquiere un derrotante performativo que refuta, un derrotante que cuenta en contra de que tenga éxito en una actuación en la que dispara y da en uno de los blancos en el campo en estas condiciones. Sin embargo, podría decirse

\footnotetext{
${ }^{14}$ También es posible modelar a los derrotantes psicológicos, tanto los que refutan como los que socavan, como derrotantes normativos. Los derrotantes normativos ejercen su función no en virtud de ser estados psicológicos del sujeto, pero en virtud de que son creencias o dudas que uno debería tener (desde un punto de vista epistémico), independientemente de si uno tiene o no estos estados psicológicos. Se puede encontrar un compendio útil de las diferencias entre estos tipos de derrotantes en Lackey (2014).
} 
que esta creencia no indica que si el disparo de la cazadora fuese exitoso, entonces no sería apto. Un disparo exitoso como éste seguramente será acreditable en gran parte a la impresionante competencia de la cazadora. ${ }^{15}$

Los derrotantes performativos, tanto los que refutan como los que socavan, también pueden darse por separado en la otra dirección, cuando un agente se encuentra en posesión de un derrotante performativo que socava, pero no uno que refuta. La cantante Adele Adkins por ejemplo, reporta una experiencia de abatimiento antes de subirse a un escenario: aunque no tiene ninguna duda sobre cómo cantar y sobre qué notas debe cantar — no es que se apresure verificando en el último momento los versos o que se preocupe de manera irracional de que va a estar demasiado ronca para cantar - ella reporta que se preocupa, de una manera nebulosa (quizás irracionalmente), de que ella va a arruinar la presentación. Hay que enfatizar que Adele no reporta ninguna creencia de que no sepa cómo cantar algunas notas o alguna canción particulares. En su lugar tiene dudas sobre sí misma - viz., sobre si su interpretación de una lista de canciones en el escenario manifestará su competencia mélica_- De esta manera un derrotante performativo que socava puede darse en ausencia de un derrotante performativo que refuta.

Con esta manera de ver los derrotantes performativos en mente, ahora podemos diagnosticar de una manera guiada por principios las diferencias principales entre el jugador de baloncesto de Sosa, quien es ignorante en el segundo orden, y la cazadora, quien está equivocada en el segundo orden, quienes (de la misma manera que el sujeto que adivina de manera fiable en EXAMEN DE LA VISTA) actúan de manera apta en el primer orden pero, en ausencia de una competencia reflexiva de segundo orden, no actúan de manera completamente apta. La diferencia es ésta: el jugador de baloncesto que es ignorante en el segundo orden no está en posesión de un derrotante performativo (del tipo que refuta o del tipo que socava), ${ }^{16}$ pero la cazadora sí lo tiene. La cazadora

${ }^{15}$ Puede encontrarse apoyo establecido a favor de esta línea de pensamiento. Véase Haddock, Millar y Pritchard (2010 capítulos 1-3); Pritchard (2012); Kallestrup y Pritchard (2012); Kallestrup y Pritchard (2013); Kallestrup y Pritchard (2014); Carter y Pritchard (2015a); Carter y Pritchard (2015b).

${ }^{16} \mathrm{Al}$ menos, la cazadora está en posesión de un derrotante performativo, un derrotante performativo que socava, del cual carece el jugador de baloncesto que es ignorante en el segundo orden. Este punto se mantiene independientemente de la cuestión de si alguno de los dos se encuentra en posesión de un derrotante performativo. Para los propósitos presentes, y para evitar algo que me parece una complicación innecesaria en vista de la cuestión que importa aquí, permanezco neutral con respecto a si debemos considerar que el jugador de baloncesto que es ignorante en el segundo orden (o Diana) se encuentra en posesión de un derrotante normativo. 
al menos se encuentra en posesión de un derrotante performativo que socava; dada la manera en la que Sosa describe el caso, es plausible que también se encuentre en posesión de un derrotante performativo que refuta ${ }^{17}$. Este es el caso incluso cuando ambos son similares con respecto a la aptitud de sus actuaciones, aunque no son competentemente reflexivos y, por lo tanto, no son completamente aptos.

Ahora bien, me parece que es importante para determinar si Sosa está en lo correcto con respecto a que el sujeto que adivina fiablemente en EXAMEN DE LA VISTA posee conocimiento animal subdóxico, determinar a su vez si el sujeto que adivina fiablemente se parece más al jugador de baloncesto (quien es ignorante en el segundo orden y quien no se encuentra en posesión de derrotantes performativos) o a la cazadora (quien está equivocada en el segundo orden y quien se encuentra en posesión de un derrotante performativo). En la siguiente sección, voy a argumentar que el sujeto que adivina de manera fiable es de hecho similar a la cazadora, no al jugador de baloncesto, vis-a-vis la derrotabilidad performativa. Sugeriré que esto as así a causa de la manera particular en la que la adivinación, entendida como una actitud representacional distinta de la creencia ordinaria, debe entenderse, suponiendo que tiene una finalidad alética.

\section{LA ADIVINACIÓN, LA VERDAD COMO FINALIDAD Y LA DERROTABILIDAD}

Un tema familiar a partir del trabajo de William James (1897) es que la mejor manera de entender la meta de la posesión de la verdad es como si fuera una mezcla de dos metas que compiten entre sí; la representación verídica y la evasión de la representación falsa. ${ }^{18}$

\footnotetext{
${ }^{17}$ Es la subestimación explícita de la cazadora de su propia proeza lo que constituye para ella un derrotante performativo que socava, en el sentido descrito. Esto es compatible con que también se encuentre en posesión de un derrotante performativo que refuta, lo cual sería el caso si ella creyese (o al menos dudase) que de hecho logró dispararle al conejo. La discusión de Sosa sobe este caso indica que Diana probablemente también tiene estas dudas, aunque esto no es hecho explícito. Es particularmente relevante que en la comparación entre Diana y el jugador de baloncesto, este último no se encuentra en posesión de ningún tipo de derrotante performativo, en virtud de de su ignorancia de segundo orden, mientras que Diana se encuentra al menos en posesión de un derrotante performativo que socava.

${ }^{18}$ Tal y como lo pone James (1987): 'hay dos maneras en las que podemos ver nuestro deber con respecto a la opinión [...] Debemos conocer la verdad. Y debemos de evitar el error —estos son nuestros mandamientos primarios [...]' Puede encontrarse una expresión contemporánea de esta idea en Alston (1985); Foley (1987); David (2001); Fallis (2006). Véase también Carter, Jarvis y Rubin (2015, y de próxima aparición).
} 
Esto sería relativamente poco interesante si estas metas no estuvieran en ninguna manera en competencia la una con la otra. ${ }^{19}$

Sin embargo, hay un sentido importante en el que la representación verídica y no representar falsamente son metas rivales. Este punto puede presentarse en términos del riesgo: uno sólo puede cumplir con la primera meta poniéndose a sí mismo en riesgo de no cumplir con la segunda. Darle más peso a la segunda meta, no representar falsamente, llevaría a uno a ser más cauto con la finalidad de evitar una posible representación falsa. De manera correspondiente, darle más peso a la primera meta tendría como resultado que uno sería más atrevido con la finalidad de poseer más verdades.

En Carter, Jarvis y Rubin (de próxima aparición), mostramos cómo distintas maneras de mezclar estas dos metas corresponden con las actitudes dóxicas en distintos lugares de la jerarquía de las actitudes representacionales. Éste es un breve compendio de esta idea:

Hay actitudes como estar seguro, en la cual se da más peso a la segunda meta (de manera que la representación falsa es peor y la falta de representación verídica no es tan mala), y hay actitudes como la sospecha, en las cuales se da más peso a la primera meta (de manera que la falta de representación verídica es peor y la representación falsa no está tan mal). Pero, la meta de todas estas actitudes dóxicas es la de poseer la verdad mediante la actitud relevante. Lo que varía es sólo la mezcla de las metas gemelas —qué tan importantes deberían ser en distintas situaciones.

Adivinar es muy parecido a sospechar ${ }^{20}$ en el siguiente sentido: ambas son actitudes que, al adoptarlas, apuntamos a la verdad, pero en un sentido más débil, o menos cauto, que en el caso de la creencia. ${ }^{21}$ Con estados como el de la sospecha y

\footnotetext{
${ }^{19} \mathrm{O}$ — usando la terminología de Martin Smith (2014) — si estas metas coincidiesen normativamente - viz., si fuesen tal que uno no podría intentar conseguir la una sin intentar automáticamente conseguir la otra $(2014,273)$.

20 Sosa, en el capítulo 8, vuelve a considerar el caso del examen de la vista, y en su discusión nota que sospechar y suponer son tipos de adivinación, adivinaciones sin asentimiento. No es claro para mí que sospechar, de acuerdo con el modelo presentado en Carter et al. (de próxima aparición), debería entenderse como un intento de dar con la verdad que no involucra asentimiento, o en su lugar, como una manera de asentir que difiere del asentimiento ordinario (basado en la creencia) en cuanto al peso que otorga a las metas gemelas de la representación verídica y no representar falsamente.

${ }^{21}$ De acuerdo con el modelo de Sosa, hay dos variedades primarias de la creencia: creencias funcionales y juicios. La discusión de la creencia en esta sección, y con referencia al modelo presentado en Carter et al. (de próxima aparición) corresponde a la creencia, per se.
} 
la adivinación, se toleran mayores posibilidades de error con tal de tener una oportunidad de poseer la verdad. ${ }^{22}$ No voy a repetir nuestro argumento a favor de esta postura aquí. ${ }^{23}$ Sin embargo, en la medida en la que el modelo propuesto es plausible — viz., que en adoptar la actitud de la sospecha, o la adivinación, intentamos dar con la verdad de una manera que se otorga un mayor peso a la representación verdadera que a evadir la representación falsa (y viceversa para la actitud de estar seguro de algo) - tenemos razón para preguntarnos si el 'conocimiento' (animal o de otro tipo) es realmente como deberíamos denominar a los logros que son el resultado de la adivinación fiable o de la sospecha.

Para apreciar este punto, hay que contrastar al sujeto que adivina fiablemente de Sosa con una leve variante de EXAMEN DE LA VISTA, la cual involucra a un creyente fiable. Supongamos que la competencia perceptivo-visual de primer orden del creyente fiable y del sujeto que adivina fiablemente es idéntica. Ambos tienen una mayor facilidad para realizar afirmaciones sobre las letras de la parte superior. El sujeto que adivina fiablemente realiza sus afirmaciones apelando a la adivinación en las filas inferiores, mientras que el creyente fiable realiza sus declaraciones apelando a creencias. ¿Cuál es la diferencia entre los dos $?^{24}$ Desde luego, decir que no

\footnotetext{
${ }^{22}$ Tal y como fue sugerido, la representación falsa causada por el engaño de un genio maligno cartesiano es un ejemplo de una posibilidad de error que es tolerable para la creencia, pero no para la actitud de estar absolutamente seguro. En el modelo presentado por Carter et al. (de próxima aparición, \$4) sugerimos que, al tolerar todas las posibilidades de error, uno da peso máximo a la representación verídica, mientras que al no tolerar ninguna posibilidad de error, uno da todo el peso a la evasión de la representación falsa.

${ }^{23}$ Véase también Carter et al. (2015 \$3).

${ }^{24}$ Es necesario considerar aquí una diferencia que se observa tanto entre la adivinación y la creencia, así como la adivinación y la estimación. La diferencia es, desde mi punto de vista, una cuestión de si el individuo toma el riesgo de poseer la verdad directa o indirectamente. El sujeto que adivina toma el riesgo de poseer la verdad de manera directa al realizar su afirmación. Pero al hacer una estimación, uno hace su afirmación con la finalidad de estar cerca de la verdad, donde estar cerca es la meta inmediata, mientras que la verdad es la meta distal. Hay precedente filosófico para considerar que la estimación corresponde a una facultad distintiva, diferente de las facultades de formación de creencias. El filósofo medieval Ibn Sina (Avicena) defendió tal facultad distintiva estimativa con la descripción de wahm. Sin embargo, su caracterización del wahm deja lugar a la sospecha de si tenía en mente una facultad que es más bien emotiva en lugar de cognitiva. Un ejemplo que Avicena utiliza frecuentemente involucra un juicio estimativo de hostilidad, del tipo que uno experimenta cuando un lobo se encuentra cerca. En algunos pasajes, Avicena divorcia explícitamente el wabm de lo que él llama el intelecto. Véase Avicena (1027 [1952]).
} 
hay ninguna diferencia no es una opción viable. ${ }^{25}$ Pero, la manera más viable de dar cuenta de cuál es la diferencia estará en última instancia en tensión con el diagnóstico de Sosa del logro del sujeto que adivina de manera fiable como conocimiento animal (subdóxico o de otro tipo). A continuación explicaré por qué.

En primer lugar, hay que considerar que es mejor entender al creyente fiable como si estuviera a la par con el jugador de baloncesto de Sosa. Hay que recordar que el jugador de baloncesto, quien encesta la pelota desde una distancia que lo pone apenas por encima del umbral de fiabilidad suficiente, pero que no se da cuenta de esto, es ignorante en el segundo orden, no es que esté equivocado en el segundo orden, y esta es la caracterización más sutil de por qué el jugador carece de la competencia reflexiva. Descrito de esta manera, dijimos que el jugador de baloncesto de Sosa no se encontraba en posesión de un derrotante performativo, como sí lo estaba la cazadora. Lo mismo sucede para una variación del sujeto que adivina de manera fiable, pero que tiene actitudes de creencia. Es decir, mantengamos fija la competencia de primer orden del sujeto que adivina fiablemente de Sosa cuando hace sus afirmaciones con respecto a las letras en la parte inferior - en cuyo caso las letras aún parecen un poco borrosas, a pesar de que las afirmaciones del sujeto son correctas - pero supongamos que la actitud tomada por parte del sujeto es una de creencia en lugar de adivinación. En tal caso nos encontramos en la misma posición que en el caso del jugador de baloncesto: tenemos una actuación apta de primer orden que no es competentemente reflexiva debido a que la actuación es ignorante en el segundo orden.

Pero el sujeto que adivina fiablemente de Sosa no es ignorante en el segundo orden, como sí lo sería si adoptara la actitud de la creencia. Y aquí me parece que (i) el modelo jerárquico de las actitudes representacionales como una función de la mezcla de las metas rivales de representar verídicamente y no representar falsamente, junto con (ii) la noción de derrotantes performativos introducida en $\$ 1$ puede ayudarnos a diagnosticar precisamente por qué.

En este momento será útil, inicialmente, considerar el caso clásico del sexador de pollos. ${ }^{26}$ Este es un caso paradigmático de un individuo que tiene una compe-

\footnotetext{
${ }^{25}$ Sosa se refiere al conocimiento animal subdóxico como "si estuviera incluso debajo del conocimiento animal que requiere de una creencia" $(2015,76)$, expresando así su postura de que el conocimiento animal subdóxico no es un tipo de conocimiento animal dóxico (basado en la creencia), sino conocimiento animal que surge de adoptar la actitud representacional más baja o más débil que es la adivinación.

${ }^{26}$ Véase, por ejemplo, Goldman (1975).
} 
tencia fiable, pero que no es competentemente reflexivo debido a que es ignorante en el segundo orden. El sexador de pollos pretende creer de cada pollo, cuál es su sexo — el sexador de pollos no te diría que está adivinando- . El sexador de pollos adopta una creencia. Esto así incluso si el sexador de pollos no puede darte ninguna razón a favor de su creencia. Tal y como Sosa $(2015,76)$ sugiere correctamente, el sexador de pollos obtiene una creencia apta, y por lo tanto conocimiento animal.

Consideremos ahora una variación en el caso del sexador de pollos. Supongamos que el sexador de pollos se dispone a adoptar la estrategia de adivinar, en lugar de adoptar creencias. Supongamos que te dice que está adivinando. ¿Consigue en este caso conocimiento animal, bajo el supuesto de que la competencia fiable de primer orden (de agrupar a los pollos por su sexo) es la misma que en el caso original? Bajo el supuesto de que es sincera, la respuesta debería ser que no, y la explicación de esto tiene dos partes. Es verosímil que el sexador de pollos, al considerar que él mismo está adivinando, haya adquirido un derrotante psicológico del cual carece el sexador de pollos que forma creencias. En efecto, a menos que uno adivine al mismo tiempo que considera que uno está adoptando creencias, uno adquiere, al recurrir a la adivinación, un derrotante psicológico — a saber, que está adivinando- lo cual socava (a menos que ya esté derrotado) el conocimiento animal de uno.

¿Por qué sucede esto? ¿Por qué la mera consciencia de que la manera en la que uno apunta hacia la verdad es una adivinación, es por sí mismo suficiente para ser un derrotante, uno que es capaz de derrotar el conocimiento animal? La respuesta es que darse cuenta de que uno está adivinando es lo mismo que darse cuenta de que uno está tratando de dar con la verdad de una manera que tolera más posibilidades de error de las que son toleradas de manera verosímil por el conocimiento. ${ }^{27}$ Sin lugar a dudas las adivinaciones pueden ser aptas. Pero cuando lo son, el logro adquirido no es lo suficientemente fiable para calificar como conocimiento, animal o de otro tipo, y eso se debe al tipo de intento intrépido de alcanzar la verdad que es una adivinanza, esto es uno que da mucho mayor peso a la representación verídica que a no representar falsamente. ${ }^{28}$

\footnotetext{
${ }^{27}$ Un corolario de este punto es que, al tratar de alcanzar la verdad mediante una adivinación, uno trata de alcanzar la verdad en ausencia de la confianza normal que se posee por default (véase Sosa 2015, 81).

${ }^{28}$ La discusión precedente es compatible con conceder que las creencias y las adivinaciones pueden no diferir en su fiabilidad. Sin embargo, como señaló un árbitro anónimo de Synthese, en algunos casos, adoptar la creencia de que estás adivinando podría de hecho tener la
} 
El análogo del jugador de baloncesto performativo al sexador de pollos cum adivinador, y también análogo al sujeto que adivina fiablemente de Sosa será un jugador que se considera a sí mismo como intentando encestar una canasta de una manera tal que tolera una posibilidad de error mayor al promedio - mediante un lanzamiento que consiste en arrojar la pelota sin cuidado- que en circunstancias normales. Inclusive si, por estipulación, el jugador de baloncesto que arroja la pelota sin cuidado es de hecho suficientemente fiable, el hecho de que el jugador de baloncesto se considere a sí mismo como arrojando la pelota sin cuidado — un método que considera menos fiable de lo usual —en lugar de lanzar la pelota de manera normal, el jugador adquiere un derrotante performativo que pone al jugador a la par con el sexador de pollos cum adivinador - a saber que ambos tratan de alcanzar la verdad mediante la adivinación, y por lo tanto tolerando un error mayor de lo normal.

El sujeto que adivina de manera fiable de Sosa en EXAMEN DE LA VISTA se encuentra a la par en términos performativos con los siguientes tres individuos que son reflexivamente incompetentes: el sexador de pollos cum adivinador y el jugador de baloncesto que adquiere un derrotante performativo gracias a que se da cuenta de que lanza la pelota sin cuidado, ${ }^{29} \sin$ darse cuenta, además, de que lo hace de manera fiable, y Diana, quien está equivocada en el segundo orden al subestimar su destreza desde el rango del que realiza el tiro. Ninguno de estos personajes es ignorante en el segundo orden; cada uno está equivocado en el segundo orden de una manera que genera un derrotante performativo. El jugador que lanza la pelota sin cuidado está equivocado porque se considera a sí mismo como si estuviera

consecuencia de reducir la fiabilidad propia en el dominio relevante, quizás de manera sustancial. Esto es así porque considerar que uno mismo está tomando niveles de riesgo mayores de los normales, podría generar, para uno, un efecto de confirmación conductual, donde el individuo incrementa los niveles de riesgo tomados a través del tiempo, volviendo sus actuaciones dóxicas (en el dominio relevante) menos conducente a la verdad, y por debajo del umbral de fiabilidad requerido para que haya aptitud de primer orden. Sin embargo, incluso si tal efecto de confirmación conductual no se materializa, el que uno se considere a sí mismo en cierta ocasión como realizando afirmaciones que involucran niveles de riesgo mayores de lo normal es en sí mismo suficiente para generar un derrotante para el potencial conocimiento del agente.

${ }^{29}$ Nótese que no se supone que este caso sea el mismo que el caso en el que uno lanza la pelota sin cuidado desde medio campo. El último es entendido mejor como un análogo a una adivinación improbable, de la cual diré algo más adelante. En su lugar, el caso al que se hace referencia antes es uno en el que se nos pide que imaginemos que el jugador, desde un punto que se encuentra apenas dentro de su umbral de fiabilidad suficiente, intenta encestar la pelo usando un estilo de lanzamiento de pelota que no es cuidadoso, un estilo que el jugador considera que no es suficientemente fiable, a pesar de que de hecho lo es. 
lanzando un tiro de una manera que se queda corta de ser suficientemente fiable. Tanto el sexador de pollos cum adivinador y el sujeto que adivina fiablemente de Sosa están equivocados porque ambos tienen una creencia que cuenta en contra de que sus afirmaciones sean suficientemente fiables - a saber, que apuntan a la verdad mediante la adivinación, y por lo tanto con una tolerancia al error mayor de lo normal.

Hay una falta de analogía interesante entre estos tres individuos que son reflexivamente incompetentes y otros tres individuos que son reflexivamente incompetentes: el sexador de pollos (ordinario), el jugador de baloncesto de Sosa (que es inconsciente de que se encuentra apenas por encima de su umbral de fiabilidad), y el sujeto de EXAMEN DE LA VISTA si éste hubiera adquirido creencias en lugar de adivinar.

\section{Algunos Paralelos NUEVOS}

Bajo el supuesto de que la discusión en la sección previa es correcta, si hay tal cosa como el conocimiento animal subdóxico, éste no será adquirido en casos en los que un individuo se considera a sí mismo como si estuviera adivinando, sin importar qué tan fiable sea in el dominio relevante de acción. Al adivinar, la manera en que apuntamos a la verdad es una manera que le da un peso desproporcionado a la representación correcta por encima de la no representación falsa, y como tal se caracteriza como una actitud que es tal que, meramente al adoptarla, asumimos un riesgo para alcanzar la verdad que involucra tolerar un número mayor de posibilidades de error de las usuales, mayor de lo que plausiblemente tolera el conocimiento. Como tal, al considerarse a uno mismo como alguien que adivina —esto es, cuando uno adivina y no se equivoca al pensar que uno está adivinando- uno adquiere de esta manera un derrotante que uno tendría que derrotar a su vez para alcanzar conocimiento animal. ${ }^{30}$ Una manera en la que se puede derrotar este derro-

${ }^{30}$ Cf., sin embargo, Hetherington (2001, 155-569) para alguien que está en desacuerdo a este respecto. Según la postura de Hetheringon — que él llama gradualismo — algunas adivinaciones pueden calificar como conocimiento, aunque califican como lo que él llama "conocimiento malo", conocimiento del más bajo tipo, que sólo requiere de creencia verdadera. Sin embargo, reconoce que esa es una posición minoritaria. Otros defensores de la postura de que la creencia verdadera, así sea adquirida por una adivinación afortunada o de alguna otra manera, asegura conocimiento son Kutschera (1982), Sartwell (1992) y Menón de Tesalia, quien defendió brevemente la tesis de que el conocimiento es meramente opinión verdadera en el Menón. Véase también Foley (2012) para una defensa más sofisticada de esta postura, en la que el conocimiento es asegurado mediante una creencia verdadera en conjunción con 
tante sería si el optometrista le informa a uno que es extremadamente fiable, de manera tal que uno no se considera más a si mismo como si estuviera adivinando cuando afirma cuáles son las letras en la línea inferior, de manera tal que uno ahora cree lo que uno afirma sobre la línea inferior.

Quiero hacer tres cosas a modo de conclusión. Primero, quiero hacer algunas observaciones acerca de los logros no fiables, en segundo lugar, quiero hacer más clara la discusión sobre cómo es que al adivinar se otorga un peso mayor a la representación correcta que a no representar falsamente, en tercer lugar, quiero presentar - a partir de la postura misma de Sosa - algunos paralelos en el campo de la acción que, me parece, tienen un correlato con respecto a distintos tipos de adivinación.

\subsection{Logros no fiables}

Incluso si las adivinaciones no pueden aspirar al conocimiento, animal o de otro tipo, esto no significa que las adivinaciones no puedan ser aptas. De hecho, las creencias del sujeto que adivina fiablemente de Sosa ciertamente son aptas, en el sentido de que su corrección manifiesta una competencia fiable de primer orden.

En Carter et al. (2015) sugerimos que, tal y como hay variedades de actitudes representacionales que apuntan a la verdad de maneras distintas (que se corresponden, dados los elementos que gobiernan esos estados, con las distintas maneras en las que se otorga peso a los bienes gemelos de alcanzar la verdad y evadir el error), también hay variedades de logros cognitivos. Una ventaja de nuestro modelo es que proporciona una explicación de por qué el conocimiento, cum logro cognitivo, debe ser seguro de manera ordinaria, y esto se debe al tipo particular de intento de tener éxito que es la creencia — a saber, un intento que pone un valor adicional en evadir el error.

Algunos logos, incluyendo los logros físicos y artísticos, son desde luego altamente no-fiables, esto es que son el resultado de medios que ordinariamente no serían exitosos. Éste es un ejemplo obvio, que sugirió Turri (de próxima aparición):

PASOS DE BEBÉ: Geno tiene once meses de edad. Cada día ha adquirido una mayor confianza en su habilidad de balancearse sobre dos pies. Hoy se levantó

información adecuada. Se puede encontrar un estudio de varias posturas que se han acercado, de diferentes maneras, a la postura de que el conocimiento es creencia verdadera en el sumario del "minimalismo epistémico" de Martens (2006). Para una crítica explícita de las posturas minimalistas, véase Lycan (1994) y Kvanvig (2003, Capítulo 1). 
apoyándose en el sillón cuando un objeto en la mano de su padre llamó su atención. Inspirado, Geno se soltó y, para regodeo de sus padres, dio sus primeros pasos en dirección hacia su padre (Turri, de próxima aparición, 14).

De manera similar, considérese el caso de un alfarero:

ALFARERO: Ben produce su cerámica característica, la cual es altamente apreciada, mediante métodos que muy a menudo producen fracturas y piezas rotas. En el caso típico, para producir una pieza nueva, podría tener que hacer varios (o muchos) intentos. De manera ordinaria, las maneras en las que produce piezas de cerámica no son seguras. ${ }^{31}$

PASOS DE BEBE y ALFARERO son ejemplos de un fenómeno que quizás puede explicarse de mejor manera apelando al caso del campeón de bateo de los medias rojas, Ted Williams: podría decirse que es el mejor bateador en la historia del béisbol, y quien bateaba de manera segura sólo un $40 \%$ de las veces. Lo que sugieren PASOS DE BEBE, ALFARERO y el caso de Ted Williams es que ciertos logros no son fiables, en el sentido de que son producidos mediante métodos no seguros.

En el caso cognitivo esto es de esperarse también. Cuando, por ejemplo, Sherlock Holmes hace una adivinación educada $a^{32}$ —una que resulta ser correcta— esto constituye un logro cognitivo, uno que es acreditable a su destreza inductiva, aunque no es conocimiento, animal o de otro tipo. Esto es así incluso si la adivinación de Sherlock resulta ser tan fiable como la del sujeto que adivina fiablemente de Sosa en EXAMEN DE LA VISTA. Sherlock, tal y como el sujeto que adivina fiablemente de Sosa, asumiendo que ambos se dan cuenta de que están adivinando, adquieren un derrotante - a saber, que al intentar dar con la verdad, han tolerado, mediante su adivinación, un número de posibilidades de error mayor que en circunstancias normales, y mayor de lo que es tolerado plausiblemente por el conocimiento.

\subsection{Adivinaciones y asignación de peso}

Supongamos que una moneda justa es lanzada y en seguida es tapada. ¿Cuál es tu nivel de creencia de que la moneda ha caído en cara? Debería ser de .5. Desde luego no deberías creer que la moneda ha caído en cara. Y esto es como debería de ser; la creencia corresponde con una mezcla de dar un peso determinado a las

\footnotetext{
${ }^{31}$ Este ejemplo ha sido adaptado a partir de un o presentado en Carter et al. $(2015,1603)$.

${ }^{32}$ Voy a considerar distintos tipos de adivinación, que incluyen a la adivinación educada, con mayor detalle más adelante en esta sección.
} 
metas de creer verdaderamente y no representar falsamente que otorga un valor mayor a no representar falsamente. En pocas palabras, la situación es más arriesgada de lo que corresponde a la creencia. ${ }^{33}$

¿Pero adivinas que la moneda ha caído en cara? Quizás sólo si hay alguna presión práctica para que hagas una afirmación. Nada de lo que sabes favorece la opción de cara sobre la de cruz. Pero puedes, sin embargo, en esta circunstancia, hacer un intento temerario de poseer la verdad - y si resulta ser cara, entonces estarías en lo correcto.

Llamemos a la actitud representacional correspondiente —una que da un peso máximo a la representación verdadera y no otorga ningún peso a no representar falsamente — una adivinación ciega. ${ }^{34} \mathrm{El}$ análogo práctico, en baloncesto, sería el caso en el que se lanza la pelota, sin ninguna apreciación de la orientación propia con respecto a la canasta - viz., un lanzamiento en la oscuridad total. Este es un intento de acertar que tolera casi todas las posibilidades de error- $-{ }^{35}$ Es adecuado que en ciertas ocasiones sea posible referirse a las adivinaciones fiables como lanzamientos en la oscuridad.

Pero no todas las adivinaciones son ciegas. El sujeto que adivina fiablemente de Sosa no es después de todo completamente insensible a la meta de no representar de manera falsa, incluso si considera que él mismo está adivinando. Concedamos que, durante el examen, a medida que las letras se vuelven más pequeñas, como Sosa describe el caso, que 'empiezo a perder la confianza de que estoy en lo correcto con respecto a cuáles son las letras'. Pero es verosímil suponer que el sujeto mantiene algo de confianza, tal que desde el punto de vista del sujeto que adivina, algunas posibilidades de error son excluidas. Tal y como sosa describe el caso, 'hay muchos casos en los que estoy bastante inseguro sobre si es una "E" o una "F",

\footnotetext{
${ }^{33}$ Es verosímil pensar que la actitud apropiada aquí es la de suspender la creencia o el juicio. Para una discusión crítica reciente sobre qué involucra esta actitud, véase Friedman (2013).

${ }^{34}$ Sosa mismo discute este tipo de adivinación bajo la descripción de adivinación total, que no es lo que lleva a cabo el sujeto que adivina fiablemente (véase Sosa 2015, 151, nota 14).

${ }^{35}$ El rango de todas las posibilidades de error que es aplicable depende en parte de la pregunta acerca de si algo ocurre, que es relevante para el caso. Por ejemplo, puedes hacer una adivinación ciega y, de esta manera, tolerar todas las posibilidades de error en un examen de opción múltiple — elige A, B C, o D— tolerando sólo tres posibilidades de error. Adivinar ciegamente cuál es el número racional que ha sido generado de manera aleatoria por una máquina, implica tolerar un número potencialmente infinito de posibilidades de error. En ambos casos, sin embargo, la adivinación ciega como tal constituye una posibilidad de poseer la verdad que acepta un riesgo máximo en el contexto de la adivinación.
} 
digamos, o una "P" en lugar de una “F”, etc.' (2015, 74). Ésta es una situación familiar: una en la que hacemos alguna afirmación, debido a la presión práctica de que lo hagamos, al tiempo de que somos conscientes de que algunas, aunque no todas, las posibilidades de error se mantienen vivas. El sujeto que adivina de Sosa no está, de manera plausible, inseguro sobre si la letra frente a él es una "I" en lugar de una "F".

La adivinación es un género que contiene diferentes especies. La adivinación, como tal, involucra tomar una posibilidad de alcanzar la verdad de una manera que mezcla las metas de representar verídicamente y no representar falsamente de una manera menos cauta que la creencia, y al hacerlo se tolera un riesgo epistémico comparativamente mayor. Las adivinaciones pueden ser más o menos cautas. Desarrollaré esta cuestión en la siguiente sección apelando a ciertas analogías con casos atléticos.

Vale la pena destacar que la caracterización precedente de la adivinación es compatible con la elucidación de Sosa de la normatividad de la adivinación — viz., como hacer una afirmación con la finalidad de 'dar con la respuesta correcta, sin hacer una afirmación con la finalidad de dar con la respuesta correcta de una manera suficientemente fiable' - incluso cuando es incompatible con la sugerencia de que la adivinación fiable aspira al conocimiento (subdóxico, animal o de otro tipo). ${ }^{36}$

\footnotetext{
${ }^{36}$ Es importante notar una potencial réplica aquí, de acuerdo con la cual los derrotantes que derrotan el conocimiento que involucra creencia (así sea animal o reflexivo) no derrotan al conocimiento animal subdóxico. Si éste fuera el caso, entonces, plausiblemente, un sujeto que adivina fiablemente podría ser un candidato a conocimiento animal subdóxico. En primer lugar, uno podría adoptar esta objeción si uno piensa que el conocimiento animal subdóxico es irrefutable —inmune a ser socavado por mecanismos ordinarios de derrota. Sin una razón basada en principios para tratar al conocimiento subdóxico de manera especial en este respecto, esta objeción no parece prometedora. Una manera ligeramente más débil de sugerir que los derrotantes que derrotan al conocimiento que involucra creencia no derrotan al conocimiento subdóxico tendría que presentar algún tipo de argumento a partir de la fuerzaque pudiera probar que los derrotantes que bastan para derrotar al conocimiento que involucra creencia no son lo suficientemente fuertes para derrotar al conocimiento animal subdóxico. Esto es más débil que la objeción considerada previamente porque no se compromete con la irrefutabilidad del conocimiento subdóxico. Sin embargo, una inspección más cercana revela que la objeción más débil parece no estar guiada por principios de una manera similar a la primera. Después de todo, en efecto sería misterioso por qué el título de 'conocimiento' es retenido en la presencia de derrotantes no derrotados (del tipo que derrotan al conocimiento que involucra creencia), incluso si algún estatus epistémico positivo fuera retenido. Agradezco a Modesto Gómez-Alonso.
} 


\subsection{Analogías performativas}

Recordemos ahora algunos paralelos performativos que hemos considerado ya: una adivinación ciega es similar a lanzar la pelota con la finalidad de hacer una canasta en condiciones en las que uno carece de cualquier apreciación sobe la orientación de uno con respecto a la canasta — viz., un lanzamiento en la oscuridad- - Un intento más cauto de alcanzar la verdad es hacer una adivinación improbable, la cual involucra hacer una afirmación con la finalidad de dar con la respuesta correcta, al mismo tiempo que se toleran más posibilidades de error que las que toma el sujeto que adivina fiablemente de Sosa, cuando el sujeto que adivina fiablemente hace una afirmación al mismo tiempo que está inseguro sobre si una letra es una "E", una "F" o una "P". En contraste, el sujeto que hace una adivinación improbable hace una afirmación (por ejemplo) al mismo tiempo que está inseguro, con respecto a una letra particular, de que (en este caso) es cualquier letra más o menos ancha, excluyendo así letras como la "I". Si el sujeto que adivina no puede ni siquiera descartar la "I", y por lo tanto hace una afirmación sin ser capaz de descartar ninguna posibilidad de error contextualmente relevante, ${ }^{37}$ la adivinación no es improbable sino ciega. En $\$ 1$ sugerí que el análogo performativo en el baloncesto de una adivinación improbable es un lanzamiento desde medio campo, bajo circunstancias normales; uno puede controlar la elección de tiro como para no hacer lanzamientos en la dirección opuesta a la canasta, algo que no puede excluirse cuando hacemos un lanzamiento en la oscuridad, al mismo tiempo que, sin embargo, se realiza el lanzamiento con el conocimiento de que se tolera la mayor parte de las posibilidades de error.

Es conveniente que demos a tales adivinaciones etiquetas tales como ciegas e improbables cuando toleran todas o la mayoría de las posibilidades de error, dado que involucran hacer una afirmación de una manera tal que no otorga ninguno o muy poco peso a la meta de no representar falsamente.

Pero hay muchas otras variedades de adivinaciones a las que es más difícil dar un nombre - aunque una manera útil de pensarlas consiste en ponerlas en yuxtaposición con sus análogos prácticos-. Específicamente, podemos hacer esto considerando los casos en los que los derrotantes performativos en casos cognitivos y no-cognitivos se alinean.

37 En el contexto de un examen de la vista, las posibilidades de error contextualmente relevantes se restringen a las letras posible. En este contexto es conocimiento común que las posibilidades de error no incluyen cosas como numerales y formas que no son letras. 
Sugerí que el sujeto que adivina fiablemente de Sosa era análogo no al jugador de baloncesto, que es ignorante en el segundo orden, quien lanza la pelota apenas por encima de su umbral de fiabilidad. Este jugador de baloncesto no está en posesión de un derrotante performativo. Pero el jugador estaría en posesión de uno si hubiese arrojado sin cuidado la pelota (en lugar de lanzarla de manera normal) de manera tal que él no supiese si es lo suficientemente fiable. Éste es el caso análogo a la adivinación fiable.

Podemos distinguir al sujeto que adivina fiablemente de Sosa de un sujeto que adivina en una situación ligeramente diferente, una distinción que se aprecia mejor considerando primero un paralelo performativo atlético. Supongamos en primer lugar que el jugador de baloncesto de Sosa se encuentra en un rango apenas por debajo de su umbral de fiabilidad suficiente, pero el jugador sabe esto, y lanza la pelota (de manera exitosa) a pesar de esto. Este caso es similar, pero no completamente, al caso del individuo que lanza la pelota sin cuidado, de una manera fiable sin que él lo sepa. Es similar en tanto que ambos se consideran a sí mismos como si estuvieran tolerando un riesgo superior al normal (el primero porque considera que lanzar la pelota sin cuidado no es lo suficientemente fiable, el segundo porque considera que sabe que, desde esa distancia y haciendo un lanzamiento de forma normal, se encuentra ligeramente debajo del umbral de fiabilidad suficiente). Pero los casos son diferentes en tanto que el jugador —al hacer el lanzamiento de manera normal, pero sabiendo que lo hace por debajo de su umbral de fiabilidad suficiente- de becho no es lo suficientemente fiable (a diferencia del que lanza la pelota sin cuidado y que es fiable sin saberlo), sin importar que el lanzamiento de hecho sea exitoso. Así, hacer adivinaciones fiables no puede ser el análogo de actuación cognitiva correcto.

El análogo correcto es, me parece, lo que llamamos una (mera) adivinación educada. Esto ocurre cuando uno, por ejemplo, se entera por el testimonio fiable del optometrista que la siguiente fila se encuentra (basándose en un cálculo reciente) muy cerca, pero justo por debajo, de su umbral de fiabilidad suficiente, y de hecho el individuo hace la afirmación de manera correcta, pero mediante una competencia que él sabe que es por poco no lo suficientemente fiable. ${ }^{38}$

\footnotetext{
${ }^{38}$ Esto tiene una similitud muy cercana al uso cotidiano de 'adivinación educada' en el que uno toma una oportunidad de poseer la verdad al mismo tiempo que cree que está tolerando un riesgo mayor al normal al hacerlo, aunque por debajo del nivel de riesgo normal que uno asume cuando trata de dar con la verdad mediante una adivinación improbable.
} 
Un tipo final de adivinación, diferente de aquellos considerados hasta hora, que me parece que merece atención es el análogo a un tipo de situación práctica más compleja — pero no poco común—. Considérese lo siguiente:

TIRO DE 3 PUNTOS: Steph, un tirador de tres puntos excelente, a menudo lee sus detalladas estadísticas de tiro para determinar si es lo suficientemente fiable desde varios puntos fuera de la línea de tres puntos, para justificar sus elecciones de tomar tiros abiertos. Steph ha leído recientemente un análisis estadístico de su desempeño desde varias distancias. La fuente que consultó, http://www. fivethirtyeight.com, utiliza modelos Bayesianos diseñados por expertos, y Steph no tiene razón para dudar que la fuente es fiable. ${ }^{39}$ Steph tiene confianza en que, si su fuente dice que se encuentra por encima de su umbral de suficiente fiabilidad en un rango determinado, entonces lo está. De esta manera, con base en esta fuente, Steph cree que es lo suficientemente fiable desde 28 pies como para justificar tomar un lanzamiento abierto a 28 pies de la canasta. Sin embargo, el entrenador de Steph también leyó el artículo, y le ha dicho a Steph antes del juego de hoy, que está seguro de que el número era 27 en lugar de 28. Steph está casi seguro de que su entrenador está equivocado y sigue creyendo que el número correcto es 28 - sin embargo, a la luz de que es incapaz (estando en la cancha) de descartar esta posibilidad de error, Steph acepta que no sabe que es lo suficientemente fiable desde 28 pies, a pesar que sigue creyendo que lo es-. Lanza la pelota desde 28 pies y la encesta.

Este caso es distinto a cualquiera de los otros que se han presentado hasta ahora. En primer lugar, tal y como el sujeto que adivina fiablemente, Steph es lo suficientemente fiable desde 28 pies. (Supongamos que el entrenador de Steph estaba equivocado). Pero, a diferencia del sujeto que adivinar fiablemente, Steph cree que es lo suficientemente fiable, a pesar de que (a la luz del testimonio equivocado de su entrenador) cree que no sabe esto. De la misma manera que el sujeto que hace una adivinación educada, Steph ha buscado información fiable independiente sobre su rango de fiabilidad. A diferencia del sujeto que hace una adivinación educada, Steph cree, antes de lanzar la pelota, que es lo suficientemente fiable desde esa distancia.

¿Tiene Steph un derrotante performativo, en el sentido que se articuló este término en $\$ 2$ ? Sí. La creencia que adquiere en la cancha, que su entrenador insiste en que el artículo decía que su umbral de fiabilidad suficiente es de 27 y no de 28 pies, cuenta en contra de la fiabilidad de la competencia de Steph desde los 28 pies, indicando que si Steph tuviese éxito desde los 28 pies, el éxito no sería

\footnotetext{
${ }^{39} \mathrm{http}$ // fivethirtyeight.com/features/sephen-currys-bombs-are-too-good-to-be-true/
} 
apto. Steph, se ha estipulado, tiene dudas acerca de la corrección de su derrotante, pero - mientras se encuentra en la cancha - no se considera a sí mismo capaz de descartarlo, de derrotar al derrotante, y de esta manera no considera que sepa que es lo suficientemente fiable desde ese rango, a pesar de que cree que lo es.

¿Qué especie de adivinación será el paralelo cognitivo de TIRO DE 3 PUNTOS? Para dar con la respuesta correcta, tratemos de modelar el tipo de derrotante performativo que Steph ha adquirido de una manera tan similar como podamos en el caso cognitivo:

PI: Elodin, un tahúr, intenta memorizar $\pi$ hasta por lo menos un dígito más que cualquiera de sus amigos, sin cometer ningún error (un solo error vuelve nula la recitación, lo cual lo descalifica de ganar la apuesta). Elodin tiene certeza cartesiana de que los primeros tres números son 3.14. Más allá de esto, su confianza se desvanece gradualmente. El 'record de Elodin, en la práctica, es de 60 dígitos antes de cometer un error. Pero, después de practicar muchas veces, ha concluido que es lo suficientemente fiable, en el contexto de la apuesta, como para intentar recitar $\pi$ hasta los 40 dígitos. El cuadragésimo dígito de $\pi$ es "1". El trigésimo noveno dígito es " 7 ". 40 Antes de la apuesta, un amigo le dice que sus cálculos de su propia fiabilidad están ligeramente equivocados y que, con base en los récords de Elodin en su entrenamiento, de hecho sólo es lo suficientemente fiable como para intentar los primeros 39 dígitos, no el cuadragésimo. Elodin cree que su amigo está equivocado, pero, se da cuenta de que no puede descartar esto de manera concluyente. Elodin, creyendo - pero creyendo que no sabe- que el dígito cuadragésimo de $\pi$ es " 1 " no se detiene en el digito trigésimo noveno. Afirma, de manera correcta, que el cuadragésimo dígito es " 1 ".

Elodin afirma que "1" al mismo tiempo que cree que no sabe es lo suficientemente fiable (dado que sabe que no puede descartar que su amigo esté en lo correcto, a pesar de que cree que su amigo está equivocado) en el contexto de su afirmación. Esta afirmación es un tipo de adivinación: un intento de dar con la verdad en el que uno toma una posibilidad de dar con la verdad de una manera que tolera un número mayor de posibilidades de error que lo usual. Pero el riesgo que Elodin toma a sabiendas es marginal. Él no cree que no sea lo suficientemente fiable; meramente cree que no sabe que es lo suficientemente fiable, al mismo tiempo que cree que lo es.

Llamemos a esta especie de adivinación una afirmación conjetural. En el caso de Elodin, la duda que tiene, aunque se la considera no derrotada en el contexto de la afirmación, se da al mismo tiempo que Elodin tiene la creencia de que no es

${ }^{40}$ Los primeros 40 dígitos de $\pi$ son: 3.1415926536897932384626433832795028841971 
los suficientemente fiable. Después de todo, Elodin, así como Steph, creen sobre la base de buenas razones que son lo suficientemente fiables, y uno hace una afirmación y el otro lanza la pelota al mismo tiempo que sostienen que no saben que son suficientemente fiables. La afirmación conjetural, como otras adivinaciones no aspiran al conocimiento (animal o de otro tipo). ${ }^{41}$

\section{CONCLUSIONES}

En este artículo he mostrado por qué la postura de Sosa con respecto a la adivinación identifica de manera equivocada ciertos tipos de adivinaciones como candidatas a conocimiento. He argumentado a favor de una manera distinta de ver la adivinación, como un tipo de actitud que, dada su misma naturaleza, da lugar a un tipo de derrotante que es adquirido cuando uno toma la oportunidad de dar con la verdad tolerando niveles de riesgo por encima de los normales. La razón

\footnotetext{
${ }^{41}$ Una aplicación interesante de esta línea de argumentación tiene que ver con el fenómeno de la amenaza de estereotipo, el cual ocurre cuando individuos que pertenecen a grupos asociados con estereotipos negativos consideran que es probable que ellos mismos exhibirán un desempeño acorde al estereotipo negativo, y esta tendencia se incremente cuando uno reflexiona acerca de la propia pertenencia a un grupo estigmatizado (véase, por ejemplo, Alfano (2014)). En casos extremos de amenaza de estereotipo, un individuo que pertenece a un grupo asociado con el estereotipo de ser poco inteligentes podría, al reflexionar sobre su membresía a este grupo, adoptar positivamente la creencia de que es no fiable cognitivamente con respecto a la tarea que desempeña. Si tal individuo hace una afirmación de todos modos, su intento de poseer la verdad se encuentra con un derrotante y por lo tanto carece de conocimiento (a menos que pueda derrotar al derrotante, por ejemplo, entendiendo los mecanismos de la amenaza de estereotipo). Los efectos perniciosos de la amenaza de estereotipo se vuelven más claros cuando consideramos casos menos extremos, casos en los que nos acercamos más a afirmaciones conjeturales, tal y como es el caso de Elodin. En tales casos, supongamos que el individuo marginalizado, al considerar su membresía al grupo marginalizado, simplemente duda acerca de su fiablidad (en algún grado) al mismo tiempo que retiene su creencia de que es lo suficientemente fiable. En tales circunstancias, incluso esta creencia de un individuo (según la postura que propongo) no es candidata a conocimiento por la misma razón que la afirmación conjetural de Elodin no es una candidata a conocimiento. Ésta es una consecuencia frustrante, pero que me parece correcta, de la postura que se ha presentado aquí, una postura que pone énfasis en un caso especial de injusticia epistémica. Tal y como Alfano (2014) ha señalado, una manera de combatir esta injusticia es dando a tales individuos marginalizados la capacidad de derrotar el tipo de derrotantes que adquieren cuando son sometidos a la amenaza de estereotipo, aprendiendo cómo funciona ésta. Gracias a un árbitro anónimo de Synthese por sugerir que tratara este asunto.
} 
que presta apoyo a la postura que he defendindo apela de una manera importante a la idea Jamesiana de que la meta de poseer la verdad se entiende mejor como una mezcla de dos metas que compiten entre si - representar verídicamente y no representar falsamente - Después de poner mi acercamiento preferido a la actitud de la adivinación en el contexto más amplio de los logros no fiables, concluí distinguiendo varias especies de adivinación en relación con ciertos análogos prácticos performativos que presenté aquí.

Quiero concluir indicando que creo que el proyecto general de Sosa en Judgment and Agency es un logro enorme en epistemología. Creo que es su presentación más convincente de su versión distintiva de la epistemología de virtudes a la fecha. La meta aquí no ha sido la de poner en cuestión el proyecto general de Sosa, como tal, sino sugerir cómo — dentro de este rico proyecto- se puede desarrollar una mejor elucidación de la adivinación, una que encaja mejor con ciertas intuiciones plausibles sobre el conocimiento y la derrotabilidad epistémica.

Traducción de Alfonso Anaya

J. Adam Carter

Eidyn Research Centre. University of Edinburgh J.Adam.Carter@ed.ac.uk

\section{BIBLIOGRAFÍA}

AlFANO, Mark (2014), "Stereotype threat and Intellectual Virtue." Naturalizing Virtue, edited by Owen Flanagan \& Abrol Fairweather, 155-74. Cambridge: Cambridge University Press.

Alston, William P. (1985), “Concepts of Epistemic Justification.” The Monist 68 (1): 57-89.

AvicenNA, (1027 [1952]) Kitab Al-Najat (the Book of Salvation). Translated by F Rahman. Oxford: Oxford University Press.

Bergmann, Michael (2005), "Defeaters and Higher-Level Requirements." Philosophical Quarterly 55: 419-36.

CARTER, J. Adam, and Navarro, Jesús (2015), “The Defeasibility of Knowledge- How.” Unpublished Manuscript.

Carter, J. Adam, and Pritchard, Duncan (2015a). "Knowledge-How and Epistemic Luck." Noûs 49 (3): 440-53.

- (2015b), "Knowledge-How and Cognitive Achievement." Philosophy and Phenomenological Research 91 (1): 181-99.

CARTER, J. Adam; Jarvis, Benjamin, and Rubin, Katherine (2015), "Varieties of Cognitive Achievement." Philosophical Studies 172 (6): 181-99. 
CARTER, J. Adam, Benjamin Jarvis, and Katherine Rubin (Forthcoming), "Belief Without Credence." Synthese, 1-29. doi:10.1007/s11229-015-0846-6.

DAVID, Marian (2001), “Truth as the Epistemic Goal." In Knowledge, Truth and Duty: Essays on Epistemic Justification, Responsibility and Virtue, edited by Matthias Steup, 151-69. Oxford: Oxford University Press.

FALLIS, Don (2006), "Epistemic Value Theory and Social Epistemology.” Episteme 2 (3): 177-88.

FolEy, Richard (1987), The Theory of Epistemic Rationality. Cambridge, MA: Harvard University Press.

- (2012), When Is True Belief Knowledge? Princeton: Princeton University Press.

FrIEDman, Jane (2013), “Suspended Judgment.” Philosophical Studies 162 (2): 165-81.

GOLDMAN, Alvin (1975), "Innate Knowledge." In Innate Ideas, edited by Stephen P Stich, 111-20. University of California Press.

Goldman, Alvin, and Erik J. Olsson (2009), "Reliabilism and the Value of Knowledge." In Epistemic Value, edited by Adrian Haddock, Alan Millar, and Duncan Pritchard, 19-41. Oxford University Press.

HAdDock, Adrian, Alan Millar and Duncan Pritchard (2010), The Nature and Value of Knowledge: Three Investigations. Oxford University Press.

HawTHorne, John (2002), "Deeply Contingent a Priori Knowledge." Philosophy and Phenomenological Research 65 (2): 517-23.

Hetherington, Stephen (2001), Good Knowledge, Bad Knowledge: On Two Dogmas of Epistemology. Oxford: Clarendon Press.

James, William (1897), The Will to Believe. New York: Longmans, Green; Company.

KALlestrup, Jesper and Duncan Pritchard (2012), "Robust Virtue Epistemology and Epistemic Anti-Individualism.” Pacific Philosophical Quarterly 93 (1): 84-103.

- (2013), "Robust Virtue Epistemology and Epistemic Dependence." In Knowledge, Virtue, and Action: Essays on Putting Epistemic Virtues to Work, edited by Tim Henning and David P Schweikard. London: Routledge.

- (2014), "Virtue Epistemology and Epistemic Twin Earth." European Journal of Philosophy 22 (3): 335-57.

KUTSCHERA, Franz von. (1982), Grundfragen der Erkenntnistheorie. Berlin: Walter de Gruyter. Kvanvig, Jonathan L. (2003), The Value of Knowledge and the Pursuit of Understanding. Cambridge University Press.

LACKEY, Jennifer (2008), Learning from Words: Testimony as a Source of Knowledge. Oxford University Press.

LYCAN, William (1994), "Sartwell's Minimalist Analysis of Knowing." Philosophical Studies 73: 1-3.

MARTENS, David (2006), "Confidence in Unwarranted Knowledge.” Erkenntnis 65 (2): 143-64.

POLlOCK, John (1986), Contemporary Theories of Knowledge. Savage, MD: Rowman \& Littlefield Publishers. 
PRITCHARD, Duncan (2012), “Anti-Luck Virtue Epistemology.” Journal of Philosophy 109 (3): 247-79.

SARTWELL, Crispin (1992), "Why Knowledge Is Merely True Belief." The Journal of Philosophy 89 (4): 167-80.

SMITH, Martin (2014), “Knowledge, Justification and Normative Coincidence." Philosophy and Phenomenological Research 89 (2): 273-95.

SosA, Ernest (2010), Knowing Full Well. Princeton: Princeton University Press [Trad. esp. de Modesto Gómez Alonso, Con pleno conocimiento, Zarasgoza: PUZ, 2014].

- (2015), Judgment and Agency. Oxford: Oxford University Press.

SuDDUTH, Michael (2008), "Defeaters in Epistemology." Internet Encyclopedia of Philosophy. http://www.iep.utm.edu/ep-defea/.

TURri, John (Forthcoming), "Knowledge as Achievement, More or Less." En M.A. Fernandez (ed.), Performance Epistemology, Oxford: Oxford University Press. 